\title{
ACCURATE AND STABLE GRID INTERFACES FOR FINITE VOLUME METHODS
}

\author{
LARS FERM $^{1} \quad$ PER LÖTSTEDT ${ }^{*} *$ \\ ${ }^{1}$ Dept of Information Technology, Scientific Computing, Uppsala University, P. O. Box 337, \\ SE-75105 Uppsala, Sweden. email: ferm@tdb.uu.se \\ ${ }^{2}$ Dept of Information Technology, Scientific Computing, Uppsala University, P. O. Box 337, \\ SE-75105 Uppsala, Sweden.email: perl@tdb.uu.se
}

\begin{abstract}
A convection-diffusion equation is discretized by a finite volume method in two space dimensions. The grid is partitioned into blocks with jumps in the grid size at the block interfaces. Interpolation in the cells adjacent to the interfaces is necessary to be able to apply the difference stencils. Second order accuracy is achieved and the stability of the discretizations is investigated. The interface treatment is tested in the solution of the compressible Navier-Stokes equations. The conclusions from the scalar equation are valid also for these equations.
\end{abstract}

Keywords: finite volume method, second order accuracy, stability, grid refinement

AMS subject classification: $65 \mathrm{~N} 12,65 \mathrm{~N} 50$

\section{Introduction}

When a partial differential equation (PDE) is solved by a numerical method, refinements of the grid are required in parts of the computational domain for accuracy and efficiency. In this way the discretization errors are controlled and there is no need for an expensive refinement of the whole domain. In adaptive methods, the discretization and solution errors are estimated and the grid is refined or coarsened based on these estimates. Examples of adaptivity for structured grids are found in [3], [8], [12]. The grids are refined in blocks with a homogeneous grid size inside a block and with jumps at the block interfaces. There are missing values at the block boundaries for the approximation of the derivatives. These values have to be computed by interpolation. The interpolation is crucial for the accuracy of the solution and the stability of the iterations to reach steady

*Financial support has been obtained from the Swedish National Network for Applied Mathematics and the Swedish Research Council for Engineering Science. 
state or the time integration, in particular for hyperbolic PDEs. Without proper treatment of the interface, the errors generated there may destroy the accuracy more than the refinement improves it.

The PDE is discretized by a second order accurate finite volume method on a structured grid in this paper. At the interface between two blocks with different grid size, the necessary interpolation is such that second order accuracy is maintained and the requirements for stability are investigated theoretically and experimentally. The difference in grid size of the cells at an interface is at most two. The equation in the analysis is the scalar convection-diffusion equation with little diffusion and the grid is Cartesian. The interpolation is of third order for second order accuracy in the first derivatives and of fourth order for the second derivatives. Standard finite volume methods are of second order on Cartesian grids and this is the rationale for the choice of interpolation accuracy. Centered difference and upwind approximation of the first derivative are discussed. An advantage with grid refinements in blocks is that the same interpolation formulas can be applied to all cells along the interface. If we allow refinement of separate cells, then it would be very complicated to obtain second order accuracy for second derivatives and many special cases would occur. Different interpolation schemes are derived and the conclusions from the convection-diffusion equation agree very well with experiments with the compressible Navier-Stokes equations discretized by the Jameson scheme [13].

Structured grids with patches of refinement have been used for time dependent and time independent problems in applications such as fluid flow [3],[8],[10],[12], electromagnetics [11], [14],[17],[21], and plasma physics [6],[18]. The missing values of the solution at the interfaces are determined by interpolation. The stability at the interface is analyzed in time and one space dimension in [2] and the importance of conservation in certain cases is pointed out in [16]. Reflection of waves in the interface is a problem for hyberbolic equations without dissipation e.g. Maxwell's equations. One dimensional problems are treated in [5] and interfaces in two dimensions in [4].

The paper is organized as follows. First, the finite volume discretization is defined. Then the different interpolation operators for computing the fine grid solution from the coarse grid data are described at an interface with a jump in the grid size. For some combinations of discretization and interpolation, the stability can be analyzed but in other cases the conclusions are drawn from numerical calculations. Finally, the Navier-Stokes equations are solved over a flat plate on a grid with blocks with different grid sizes. The unstable cases are the same in the nonlinear system of equations as in the study of the scalar convection-diffusion equation. 


\section{Finite volume discretization}

We wish to solve a PDE written in conservative form

$$
\frac{\partial w}{\partial t}+\frac{\partial f(w, \nabla w)}{\partial x}+\frac{\partial g(w, \nabla w)}{\partial y}=0,(x, y, t) \in \Omega \times\left(0, t_{E}\right] .
$$

The space derivatives are approximated by a finite volume method on a structured grid but the same technique is applicable to unstructured grids composed of triangles. Let $f=\cos (\theta) w-\mu w_{x}$ and $g=\sin (\theta) w-\mu w_{y}$ in (1) and let a partial derivative with respect to $x, y$, or $t$ be denoted by a subscript. Then a scalar model problem is

$$
\begin{aligned}
& w_{t}+(\cos (\theta) w)_{x}+(\sin (\theta) w)_{y}=\mu\left(w_{x x}+w_{y y}\right) \\
& x_{\text {min }} \leq x \leq x_{\text {max }}, y_{\text {min }} \leq y \leq y_{\text {max }} \\
& w\left(x, y_{\text {min }}\right)=w\left(x, y_{\text {max }}\right)
\end{aligned}
$$

The direction of the convection in (2) is given by $\theta \in[0, \pi)$ and the viscosity by $\mu \geq 0$. It is sufficient to consider $\theta \in[0, \pi)$ because of the symmetry of the problem. If $\theta \in[0, \pi / 2)$ then the boundary condition is $w\left(x_{\min }, y\right)=w_{0}(y)$ and if $\theta \in(\pi / 2, \pi]$ then $w\left(x_{\text {max }}, y\right)=w_{0}(y)$.

Integrate (1) over grid cell $\omega_{j k}$ of area $\left|\omega_{j k}\right|$ and use Gauss' theorem. Then we obtain the equation for the cell average $w_{j k}$

$$
\frac{\partial w_{j k}}{\partial t}+\frac{1}{\left|\omega_{j k}\right|} \int_{\partial \omega_{j k}}\left(f n_{x}+g n_{y}\right) d S=0,
$$

where $\partial \omega_{j k}$ is the cell boundary and $\left(n_{x}, n_{y}\right)$ is its normal. We need approximations of $f$ and $g$ on $\partial \omega_{j k}$ using the averages $w_{l m}$ from adjacent cells.

Consider our scalar model problem (2). We use two different approximations of $w$ at the cell faces. On the face $(j+1 / 2, k)$ in Fig. 1 between the cells $(j, k)$ and $(j+1, k)$, either $w$ is approximated by

$$
w_{j+1 / 2, k}=\left(w_{j k}+w_{j+1, k}\right) / 2,
$$

yielding a centered difference approximation of $w_{x}$, or

$$
w_{j+1 / 2, k}=\left(3 w_{j k}-w_{j-1, k}\right) / 2 \text { or } w_{j+1 / 2, k}=\left(3 w_{j+1, k}-w_{j+2, k}\right) / 2,
$$

(depending on $\theta$ ) giving an upwind approximation. The $y$-direction is treated similarly. The discretizations of the convective terms are second order accurate on Cartesian grids with constant step sizes $h_{x}$ and $h_{y}$ in the $x$ - and $y$-directions with (4) and (5). The stencils for the convective term $w_{x}$ on such grids are

$$
S_{c}^{c}=h^{-1}(-1 / 2,0,1 / 2), S_{u+}^{c}=h^{-1}(1 / 2,-2,3 / 2), S_{u-}^{c}=h^{-1}(-3 / 2,2,-1 / 2),
$$


for the cell in the middle of $S_{c}^{c}$, for the right cell in $S_{u+}^{c}$ if $\theta \in[0, \pi / 2)$, and for the left cell in $S_{u-}^{c}$ if $\theta \in(\pi / 2, \pi]$.

Methods for systems of conservation laws governing fluid flow are found in [20]. The scheme in [13] corresponds to a centered difference method for (2). Artificial dissipation $\eta$ of fourth order is added in the $j$ and $k$ directions for stability in subsonic flow in [13]. With the shift operator $E$, defined by $E w_{j k}=w_{j+1, k}, \eta_{j k}$ in the $j$ direction in cell $(j, k)$ is

$$
\eta_{j k}=\sigma h^{-1}\left(E^{1 / 2}-E^{-1 / 2}\right)^{4} w_{j k},
$$

where $\sigma>0$ is a parameter. The artificial dissipation in the $k$ direction is defined analogously.

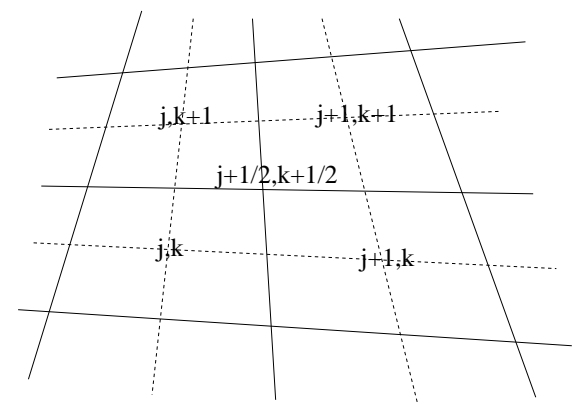

(a)

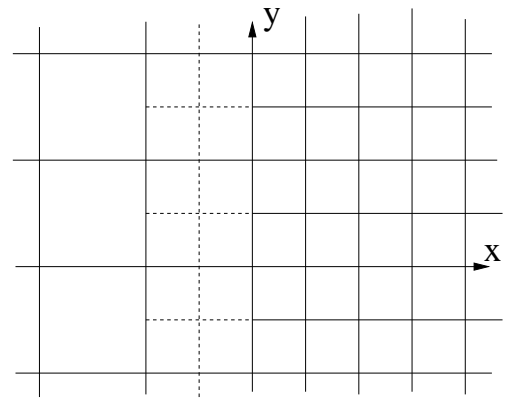

(b)

Figure 1: The primal (solid) and dual (dashed) grid (a), and a block interface at $x=0$ with the primal (solid) and the ghost (dashed) cells (b).

For calculation of the gradient $\nabla w$, a dual grid is generated by combining the centers of the cells in the primal, original grid, see Fig. 1. Then the cell average $\nabla w_{J}$ is computed in a dual cell $\omega_{J}$ with the following variant of Gauss' theorem

$$
\nabla w_{J}=\frac{1}{\left|\omega_{J}\right|} \int_{\omega_{J}} \nabla w d \omega=\frac{1}{\left|\omega_{J}\right|} \int_{\partial \omega_{J}} w n d S .
$$

At the dual face $(j+1, k+1 / 2)$ between dual cells $(j+1 / 2, k+1 / 2)$ and $(j+$ $3 / 2, k+1 / 2)$, see Fig. 1 , we take $w_{j+1, k+1 / 2}=0.5\left(w_{j+1, k}+w_{j+1, k+1}\right)$. In the same manner $w_{j+1 / 2, k+1}, w_{j, k+1 / 2}$, and $w_{j+1 / 2, k}$ are determined to compute $\nabla w_{j+1 / 2, k+1 / 2}$ with $J=(j+1 / 2, k+1 / 2)$ in (8). Then $\nabla w_{j-1 / 2, k+1 / 2}, \nabla w_{j+1 / 2, k-1 / 2}$, and $\nabla w_{j-1 / 2, k-1 / 2}$ are calculated. At the face $(j+1 / 2, k)$ in the primal grid we let $\nabla w_{j+1 / 2, k}=0.5\left(\nabla w_{j+1 / 2, k+1 / 2}+\nabla w_{j+1 / 2, k-1 / 2}\right)$ to be inserted in the flux calculation in (3). A one-sided computation of the gradient at the face $(j+1 / 2, k)$ is obtained by first computing $\nabla w_{j-1 / 2, k}$ and $\nabla w_{j-3 / 2, k}$. Then these values are extrapolated to $(j+1 / 2, k)$

$$
\nabla w_{j+1 / 2, k}=2 \nabla w_{j-1 / 2, k}-\nabla w_{j-3 / 2, k} .
$$


Only variables of $w_{l k}$ with $l \leq j$ are involved in this approximation as in (5). The second order accurate stencils for the viscous term $w_{x x}$ in a Cartesian grid with $h=h_{x}=h_{y}$ with the centered and one-sided differences, $S_{c}^{v}$ and $S_{u}^{v}$, are

$$
S_{c}^{v}=h^{-2}\left(\begin{array}{ccc}
1 / 4 & -1 / 2 & 1 / 4 \\
1 / 2 & -1 & 1 / 2 \\
1 / 4 & -1 / 2 & 1 / 4
\end{array}\right), S_{u}^{v}=h^{-2}\left(\begin{array}{cccc}
-1 / 4 & 1 & -5 / 4 & 1 / 2 \\
-1 / 2 & 2 & -5 / 2 & 1 \\
-1 / 4 & 1 & -5 / 4 & 1 / 2
\end{array}\right),
$$

for the cell in the center of $S_{c}^{v}$ and the middle cell to the right in $S_{u}^{v}$.

The computational grid is partitioned into blocks. The space discretization in cells at a block boundary is simplified if each block has two rows of ghost cells overlapping the adjacent block. A discontinuous change of the step size by at most a factor two is permitted at a block boundary. In Fig. 1.b, a block interface is depicted with the ghost cells of the fine block. The ghost cells of the coarse block consist of four fine grid cells.

\section{$3 \quad$ Accuracy and interpolation}

The variables in the ghost cells are either copied, averaged, or interpolated from the neighboring block. The value in a coarse ghost cell overlapping four fine grid cells is computed by area weighted averaging. This is an exact calculation on a Cartesian grid. If the cells are of the same size at a block boundary, then the variables in the ghost cells are copied from the adjacent block. The variables in the ghost cells in the fine grid have to be interpolated from available coarse grid values and possibly the coarse ghost cells. In this section we construct high order interpolation operators from coarse to fine blocks.

For second order accuracy on a Cartesian grid, $f$ and $g$ in (3) have to be computed with third order accuracy on a cell face at a block boundary [12]. To determine a third order accurate $\nabla w$ using (8) at a block boundary, $w$ in a ghost cell must be interpolated with fourth order accuracy. If $f$ and $g$ only depend on $w$, then third order interpolation is sufficient.

We thus want to construct interpolation operators of high order. Fig. 2. displays cells at a block interface between a block with a fine grid to the left and a coarse grid to the right. The coarse cells are marked with the cell averages $w_{i j}$ in the Figure. The interior of the coarse block is found to the right where $i \geq 1$. We derive interpolation operators for the four fine ghost cells denoted by $A, B, C, D$ which are contained in the coarse interior cell with solution average $w_{1 j}$. The interpolation operators should be applicable for all fine ghost cells along the interface. To avoid problems at block corners the interpolation stencils should not cover more than two cell layers upwards and downwards. Difficulties at block corners are also the reason why we only use two coarse ghost cells $(i \leq 0)$ in the left part of the Figure. 


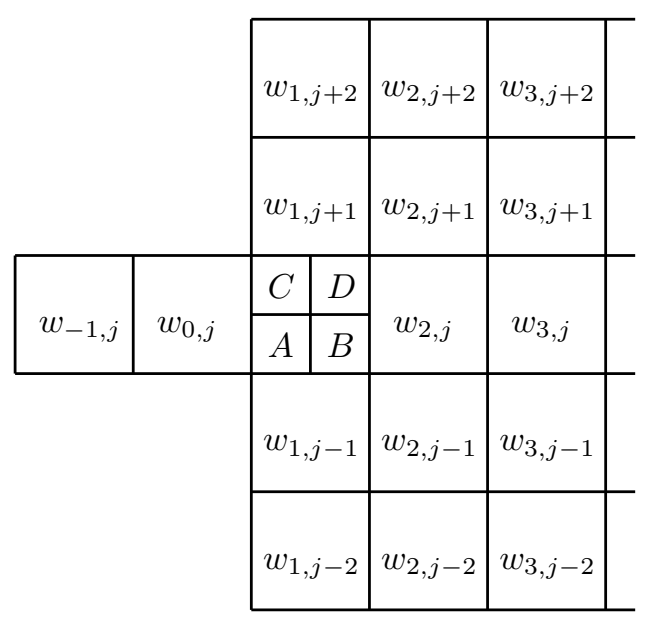

Figure 2: Cells at a block interface with a coarse block to the right. Interpolations are derived for the fine ghost cells $A-D$.

A Taylor expansion of $w$ of order three contains ten terms in 2D. We choose ten coarse cells for a fourth order accurate interpolation, integrate the terms in the Taylor expansion of $w$ over each cell and match these expansions with the expansion for one of the cells $A, B, C$, or $D$. We obtain a $10 \times 10$ linear system for the interpolation coefficients. If the matrix is non-singular, we can derive an interpolation formula for the average in the fine ghost cells. Assume that the interpolation for cell $A$ is

$$
w_{A}=w_{0}+\sum_{i} \sum_{k \neq 0} a_{i k} w_{i, j+k},
$$

where $w_{0}=\sum_{i} a_{i 0} w_{i j}$. Mirroring in the $x$-axis gives a corresponding formula for cell $C$

$$
w_{C}=w_{0}+\sum_{i} \sum_{k \neq 0} a_{i k} w_{i, j-k}
$$

The sets of interpolation cells for $A$ and $C$ are identical only if they are symmetric with respect to $k$.

Consider a solution which is constant in the direction normal to the interface. Then $w_{i j}=w_{1 j}$ for all $i, j$ and

$$
w_{A}=w_{B}=\sum_{k} a_{., k} w_{1, j+k},
$$

where $a_{., k}=\sum_{i} a_{i k}$. Thus the coefficients $a_{., k}$ define a fourth order interpolation formula in 1D. We may use it in the general case for interpolation into the double 
cell $A B$

$$
w_{A B}=\sum_{k} a_{., k} w_{1, j+k}
$$

This increases the number of involved coarse cells if $a_{1 k}=0$ but $a_{,, k} \neq 0$ for some $k$. Since $2 w_{A B}=w_{A}+w_{B}$, the interpolation

$$
w_{B}=2 \sum_{k} a_{., k} w_{1, j+k}-w_{A}=2 a_{., 0} w_{1 j}+2 \sum_{k \neq 0} a_{., k} w_{1, j+k}-w_{A}
$$

is of fourth order.

Correspondingly,

$$
w_{D}=2 a_{., 0} w_{1 j}+2 \sum_{k \neq 0} a_{., k} w_{1, j-k}-w_{C} .
$$

The use of full interpolation formulas for the four cells $A-D$ requires $4 \times 19=76$ floating point operations (flops). By the above we make use of some redundancy and reduce the work by about $40 \%$.

Consider a symmetric operator where $a_{i k}=-a_{i,-k}$. Every interpolation formula satisfies the condition $\sum_{i} \sum_{k} a_{i k}=1$, and hence $a_{r, 0}=1$ in the symmetric case since $\sum_{k \neq 0} a_{i, k}=0$. Let

$$
\begin{aligned}
& S_{0}=\sum_{i} \sum_{k \neq 0} a_{i k} w_{i, j+k}=\sum_{i} \sum_{k>0} a_{i k}\left(w_{i, j+k}-w_{i, j-k}\right), \\
& S_{1}=2 \sum_{k \neq 0} a_{., k} w_{1, j+k}-S_{0}=2 \sum_{k>0} a_{., k}\left(w_{1, k+j}-w_{1, j-k}\right)-S_{0} .
\end{aligned}
$$

Then with $w_{1}=2 w_{1 j}-w_{0}$ by (11), (12), (15), and (16)

$$
w_{A}=w_{0}+S_{0}, w_{C}=w_{0}-S_{0}, w_{B}=w_{1}+S_{1}, w_{D}=w_{1}-S_{1} .
$$

A non-symmetric operator can be symmetrized in the following way. Derive the interpolation formulas (11) and (12) for the fine ghost cells $A$ and $C$ as described above. Suppose that they are based on different sets of coarse interpolation cells. Derive a new operator for $C$ analogous to (15) for $B$, using 1D-interpolations in the normal direction for the double cell $A C$. Let

$$
w_{C}^{\prime}=2 w_{A C}-w_{A},
$$

where $w_{A C}=\sum_{i} a_{i,} w_{i j}$ and $a_{i, \cdot}=\sum_{k} a_{i k}$ (cf. $w_{A B}$ in (14)). The interpolations (12) and (17) for $C$ use different sets of coarse grid points. The average is

$$
\begin{gathered}
w_{C}^{*}=\frac{1}{2}\left(w_{C}+w_{C}^{\prime}\right)=\frac{1}{2}\left(w_{0}+\sum_{i} \sum_{k \neq 0} a_{i k} w_{i, j-k}+2 w_{A C}\right. \\
\left.-w_{0}-\sum_{i} \sum_{k \neq 0} a_{i k} w_{i, j+k}\right)=w_{A C}+\sum_{i} \sum_{k \neq 0} 0.5\left(a_{i,-k}-a_{i k}\right) w_{i j+k}
\end{gathered}
$$


which is a symmetric operator.

Since a reasonable operator is concentrated around the cells $A-D$, the expansion to a symmetric operator usually means adding just a couple of cells. The operator we have used for most computations involves 12 coarse cells, and the values at the fine ghost cells $A-D$ are calculated in just 27 flops.

A list of interpolation operators for cell $A$ is given in Table 1. Interpolations of order three and four are denoted by $L_{i}$ and $H_{i}$, respectively. The interpolation weights are multiplied by a factor 64 in the table. The indices $\left(i_{1}, k_{1}\right)$ and $\left(i_{2}, k_{2}\right)$ vary with the operator. In all cases $\left(i_{1}, k_{1}\right)=(4,0)$ except for the operators $H_{6}$ and $H_{7}$ where $\left(i_{1}, k_{1}\right)=(-1,0)$, and $\left(i_{2}, k_{2}\right)=(0,0)$ except for operator $H_{2}$ where $\left(i_{2}, k_{2}\right)=(1,2)$. The weights for the symmetric versions $L_{i}^{*}$ and $H_{i}^{*}$ of the operators are calculated as in (18). The interpolation operators that use only interior cells in the coarse grid are $L_{2}, H_{1}, H_{2}, H_{3}$. A coarse cell $(i, j+k)$ is labelled $(i, k)$ in the table.

\begin{tabular}{|c|c|c|c|c|c|c|c|c|c|c|c|c|}
\hline & $1,-1$ & 1,0 & 1,1 & $2,-1$ & 2,0 & 2,1 & $3,-1$ & 3,0 & 3,1 & $1,-2$ & $i_{1}, k_{1}$ & $i_{2}, k_{2}$ \\
\hline$L_{1}$ & 12 & 60 & -8 & -4 & -4 & 0 & 0 & 0 & 0 & 0 & 0 & 8 \\
\hline$L_{2}$ & 12 & 84 & -8 & -4 & -28 & 0 & 0 & 8 & 0 & 0 & 0 & 0 \\
\hline$H_{1}$ & 21 & 82 & -7 & -6 & -43 & 2 & 2 & 21 & 0 & -3 & -5 & 0 \\
\hline$H_{2}$ & 28 & 73 & 0 & 0 & -55 & 8 & -1 & 27 & -3 & $-11 / 2$ & -5 & $-5 / 2$ \\
\hline$H_{3}$ & 22 & 80 & -6 & -8 & -39 & 0 & 3 & 19 & 1 & -3 & -5 & 0 \\
\hline$H_{4}$ & $43 / 2$ & 61 & $-13 / 2$ & -7 & -11 & 1 & $5 / 2$ & 0 & $1 / 2$ & -3 & 0 & 5 \\
\hline$H_{5}$ & 21 & 62 & -7 & -6 & -13 & 2 & 2 & 1 & 0 & -3 & 0 & 5 \\
\hline$H_{6}$ & 21 & 56 & -7 & -6 & -9 & 2 & 2 & 0 & 0 & -3 & -1 & 9 \\
\hline$H_{7}$ & 19 & 36 & -9 & -2 & -1 & 6 & 0 & 0 & -2 & -3 & -5 & 25 \\
\hline
\end{tabular}

Table 1: Weights on coarse cells $(i, k)$ for interpolation into cell $A$ for a number of operators. The weights are multiplied by 64 .

The scalar model equation (2) is discretized with the centered stencils $S_{c}^{c}$ in (6) and $S_{c}^{v}$ in (10). The domain $\Omega=[0,2] \times[0,1]$ is partitioned into two blocks with $n \times n$ cells and grid size $h$ to the left and $2 n \times 2 n$ cells of size $h / 2$ to the right. Let $r$ denote the space operator in (2) and $r_{d}$ its discretization. Let $w=\cos (x / 4)$ and compute the difference

$$
d_{i j}=\left(r_{d} \int_{\omega_{i j}} w d \omega-\int_{\omega_{i j}} r w d \omega\right) / h_{i j}^{2}
$$

for each cell $\omega_{i j}$ of size $h_{i j}$.

The results along a normal across the interface are displayed in Fig. 3 for $n=6,12,24$. The larger errors at the block interface in the figures to the left are obtained with third order interpolation $L_{1}$ in Table 1 , and the small errors to the right are computed with fourth order interpolation $H_{7}$. Increasing error constants when the grid is refined are observed for third order interpolation when the dissipative terms dominate, indicating that the difference approximation is 
only first order accurate at the interface, see Fig. 3.a. With fourth order interpolation in Fig. 3.c the approximation is of second order everywhere. When the convection terms dominate in Figs. 3.b and 3.d third order interpolation may suffice. In Fig. 3.d we see a discretization which is close to third order accurate. The advantage of higher order interpolations is obvious in particular for larger diffusion constants $\mu$.

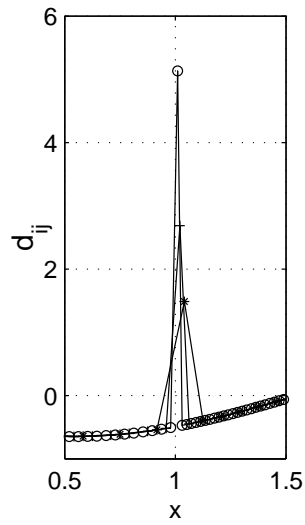

(a)

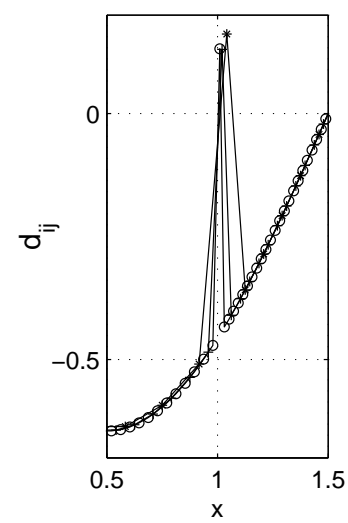

(b)

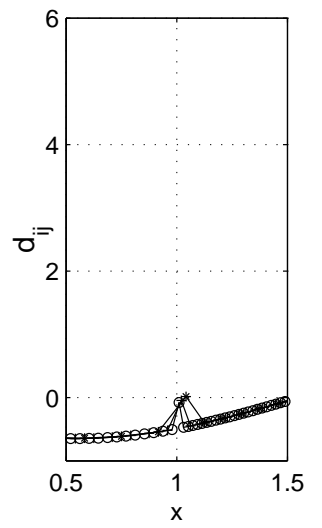

(c)

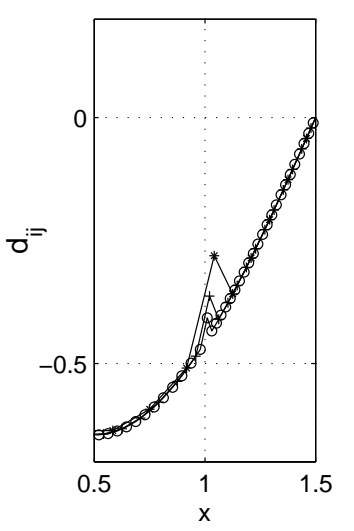

(d)

Figure 3: Discretization error constant $d$ in (18) at block interfaces for the dissipative constant $\mu=0.1$ (a,c) and $\mu=10^{-3}$ (b,d). The orders of interpolation are three $(\mathrm{a}, \mathrm{b})$ and four $(\mathrm{c}, \mathrm{d})$ and the number of cells are $6(*), 12(+)$, and 24 (o).

\section{Stability}

The stability of the space discretization and the interpolation at a block interface is investigated for the scalar model problem in this section. The equation (2) is discretized in space on a Cartesian grid, see Fig. 1. Then it can be written as

$$
W_{t}+R(\theta, \mu) W=b, W, b \in \mathbb{R}^{N}, R \in \mathbb{R}^{N \times N},
$$

where $N$ is the total number of cells and $b$ is determined by the boundary conditions. The unknown variables in $W$ are ordered with increasing index in $j$ from the left to the right in each row and from the bottom to the top row with increasing $k$ (see Fig. 1). The right block has the same ordering. At the outflow boundary at $x=x_{\min }$ or $x=x_{\max }$, we use an upwind approximation of $w_{x}$ and $w_{x x}$.

The time integration in (19) is stable in a certain sense if $R(\theta, \mu)$ is diagonalizable and the eigenvalues $\lambda_{j}$ of $R$ satisfy $\Re \lambda_{j}>0$. This statement is related to the Godunov-Ryabenkii condition for initial-boundary value problems [9]. We partition $R$ into blocks $R_{l l}$ and $R_{r r}$ corresponding to the $n_{l}$ and $n_{r}$ cells and 
unknowns in the left and right blocks, respectively, so that

$$
R=\left(\begin{array}{ll}
R_{l l} & R_{l r} \\
R_{r l} & R_{r r}
\end{array}\right), R_{l l} \in \mathbb{R}^{n_{l} \times n_{l}}, R_{r r} \in \mathbb{R}^{n_{r} \times n_{r}} .
$$

The interpolation of fine grid data to the coarse grid equations is found in submatrix $R_{l r}$ and in $R_{r l}$ the coarse grid data are transfered to the fine grid. The matrices $R_{l r}$ and $R_{r l}$ are both non-zero for the centered approximations $S_{c}^{c}$ in (6) and $S_{c}^{v}$ in (10). With upwind discretizations with stencils $S_{u+}^{c}$ in (6) and $S_{u}^{v}$ in (10) in the coarse block at the interface and a flow from the left, we have $R_{l r}=0$. If $\theta \in(\pi / 2, \pi]$ and the upwind approximations are used at least at the interface, then $R_{r l}=0$. In both these cases the eigenvalues of $R_{l l}$ and $R_{r r}$ are also eigenvalues of $R$.

We will now analyze a case when $R_{l r}=0$. Consider the case $y_{\min }=0$, $\theta \in[0, \pi / 2)$, and the discretization:

1. $w_{x}$ in (2) with the centered stencil $S_{c}^{c}$ in the interior and $S_{u+}^{c}$ at the right boundary of the block,

2. $w_{x x}$ in (2) with the centered stencil $S_{c}^{v}$ in the interior and $S_{c}^{v}$ at the right boundary.

In the $y$ direction, $w_{y}$ is approximated by the centered difference $S_{c}^{c}$ and $w_{y y}$ by the stencil $(1,-2,1)$. This is the discretization for the convection terms utilized in the inviscid calculations in [12].

The solution is Fourier expanded in the $y$ direction. Let the coarse grid have $n_{x}$ cells in the $x$ direction and $n_{y}$ in the $y$ direction. Then we obtain for each row $k$

$$
W_{j k}=w_{j}(\omega) \exp \left(i \omega y_{k}\right), y_{k}=(k-1 / 2) h, j=1, \ldots, n_{x}, k=1, \ldots, n_{y},
$$

for $n_{y}$ discrete values $\omega_{l}=2 \pi(l-1) /\left(\left(n_{y}+1 / 2\right) h\right), l=1, \ldots n_{y}$. The space discretization for the cells in one row of the left grid can be written as

$$
\begin{aligned}
& \cos (\theta)(2 h)^{-1} C w \exp \left(i \omega y_{k}\right)+i \sin (\theta) h^{-1} \sin (\omega h) w \exp \left(i \omega y_{k}\right) \\
& -\mu h^{-2} \cos (\omega h / 2)^{2} V w \exp \left(i \omega y_{k}\right)+4 \mu h^{-2} \sin (\omega h / 2)^{2} w \exp \left(i \omega y_{k}\right)-f_{k},
\end{aligned}
$$

where

$$
C=\left(\begin{array}{ccccc}
0 & 1 & & & \\
-1 & 0 & 1 & & \\
& \ddots & \ddots & \ddots & \\
& & -1 & 0 & 1 \\
& & 1 & -4 & 3
\end{array}\right), V=\left(\begin{array}{ccccc}
-2 & 1 & & & \\
1 & -2 & 1 & & \\
& \ddots & \ddots & \ddots & \\
& & 1 & -2 & 1 \\
& -1 & 4 & -5 & 2
\end{array}\right),
$$

$C, V \in \mathbb{R}^{n_{x} \times n_{x}}$, and $f_{k}$ contains the boundary terms. 
Let $\lambda_{j}(S), j=1: n_{x}$, be the eigenvalues of $S$ in

$$
S(\theta)=\cos (\theta)(2 h)^{-1} C-\mu h^{-2} \cos (\omega h / 2)^{2} V .
$$

The discrete $\omega$-values are such that $\cos (\omega h / 2) \neq 0$. Then it follows from $(22)$ that the eigenvalues of $R_{l l}$ are

$$
\begin{aligned}
& \lambda_{j k}\left(R_{l l}\right)=\lambda_{j}(S)+i \sin (\theta) h^{-1} \sin \left(\omega_{k} h\right)+4 \mu h^{-2} \sin \left(\omega_{k} h / 2\right)^{2}, \\
& j=1, \ldots n_{x}, k=1, \ldots n_{y} .
\end{aligned}
$$

The discretization in the right block is the same as in the left block. With the interpolations $L_{2}, H_{1}, H_{2}$, or $H_{3}$ in Table 1 only variables from the coarse block are used to update the fine ghost cells. Therefore, $R_{r r}$ has the same structure as $R_{l l}$ and its eigenvalues are as in (25) with $h$ replaced by $h / 2$.

If the wind blows in the opposite direction, $\theta>\pi / 2$, then the structure of $R$ is

$$
R=\left(\begin{array}{cc}
R_{l l} & R_{l r} \\
0 & R_{r r}
\end{array}\right)
$$

The eigenvalues of $R$ are still the eigenvalues of $R_{l l}$ and $R_{r r}$. Values to the right of the boundary cell now form the upwind stencils $S_{u-}^{c}$ and $S_{u}^{v}$. The centered approximations are the same. By applying the difference stencil to $W_{j k}$ in (21) we arrive at

$$
\begin{aligned}
& \cos (\theta)(2 h)^{-1} C^{*} w \exp \left(i \omega y_{k}\right)+i \sin (\theta) h^{-1} \sin (\omega h) w \exp \left(i \omega y_{k}\right) \\
& -\mu h^{-2} \cos (\omega h / 2)^{2} V^{*} w \exp \left(i \omega y_{k}\right)+4 \mu h^{-2} \sin (\omega h / 2)^{2} w \exp \left(i \omega y_{k}\right)-f_{k} .
\end{aligned}
$$

Here, $C^{*}$ and $V^{*}$ are permutations of $-C$ and $V$

$$
C^{*}=-Z C Z, V^{*}=Z V Z, Z_{j k}=\delta_{j, n_{x}+1-k}, j, k=1: n_{x},
$$

where $\delta_{j k}$ is the Kronecker tensor. The eigenvalues of $C^{*}$ and $V^{*}$ are

$$
\lambda_{j}\left(C^{*}\right)=-\lambda_{j}(C), \lambda_{j}\left(V^{*}\right)=\lambda_{j}(V), j=1: n_{x},
$$

and as in (25)

$$
\begin{aligned}
& \lambda_{j k}\left(R_{l l}\right)=\lambda_{j}\left(S^{*}\right)+i \sin (\theta) h^{-1} \sin \left(\omega_{k} h\right)+4 \mu h^{-2} \sin \left(\omega_{k} h / 2\right)^{2}, \\
& S^{*}(\theta)=\cos (\theta)(2 h)^{-1} C^{*}-\mu h^{-2} \cos (\omega h / 2)^{2} V^{*} .
\end{aligned}
$$

Let $\phi=\pi-\theta$ for $\theta \in(\pi / 2, \pi]$ in (30). Then by (28), (25), and (24) we infer that

$$
S^{*}(\theta)=-\cos (\theta)(2 h)^{-1} Z C Z-\mu \cos (\omega h / 2)^{2} h^{-2} Z V Z=Z S(\phi) Z,
$$

and the spectra of $S^{*}(\theta)$ and $S(\phi)$ are the same. In the intermediate case when $\theta=\pi / 2$, the factor in front of $C$ and $C^{*}$ in (24) and (30) vanishes. 
For stability we require $\Re \lambda(R)>0$ for $R$ in (19). It follows from (20) and (26) that the eigenvalues of $R$ are the eigenvalues of $R_{l l}$ and $R_{r r}$. Their eigenvalues are given by (25) and (30). The conclusion is that the discretization is stable if $\Re \lambda_{j}(S)>0$ for all $j$ and $\theta \in[0, \pi / 2)$.

In order to study the stability of $C$ and $V$ in (23) ( and $S$ when $\mu=0$ and $\theta=\pi / 2$ ) we derive a lemma for the eigenvalues of a general matrix $A$ of the form

$$
A=\left(\begin{array}{cccccc}
\beta & \gamma & & & & \\
\alpha & \beta & \gamma & & & \\
& & \ddots & \ddots & \ddots & \\
& & & \alpha & \beta & \gamma \\
0 & \kappa_{m} & \cdots & \kappa_{2} & \kappa_{1} & \kappa_{0}
\end{array}\right) .
$$

With the lemma we can bound the real part of the eigenvalues of $C$ and $V$ in a theorem. The proofs are inspired by similar results in [15].

Lemma 4.1 Assume that $\alpha \neq 0, \gamma \neq 0, \kappa_{m} \neq 0$, in (31) and let $\xi_{j}, j=$ $1, \ldots m+1$, be the zeros of

$$
g(\xi)=-\xi^{m+1}+a_{0} \xi^{m}+\left(a_{1}-1\right) \xi^{m-1}+\sum_{j=2}^{m} a_{j} \xi^{m-j}=\prod_{j=1}^{m+1}\left(\xi-\xi_{j}\right),
$$

where $a_{0}=\left(\kappa_{0}-\beta\right) / \sqrt{\alpha \gamma}, a_{j}=\kappa_{j} /\left(\rho^{j} \sqrt{\alpha \gamma}\right), j=1, \ldots m$, and $\rho=\sqrt{\alpha / \gamma}$. The eigenvalues of $A$ in (31) satisfy

$$
\lambda(A)=\beta+\sqrt{\alpha \gamma}\left(\xi_{*}+\xi_{*}^{-1}\right),
$$

where $\xi_{*}$ is a root of

$$
\xi^{2 n-m+1} \prod_{j=1}^{m+1}\left(\xi-\xi_{j}\right)=(-1)^{m+1} \prod_{j=1}^{m+1} \xi_{j} \prod_{j=1}^{m+1}\left(\xi-\xi_{j}^{-1}\right) .
$$

Proof Let $D$ be a diagonal matrix with the elements $D_{j j}=\rho^{j-1}, j=1, \ldots n$ and let

$$
B=\left(\begin{array}{cccccc}
0 & 1 & & & & \\
1 & 0 & 1 & & & \\
& & \ddots & \ddots & \ddots & \\
& & & 1 & 0 & 1 \\
0 & a_{m} & \cdots & a_{2} & a_{1} & a_{0}
\end{array}\right)
$$

Then

$$
D^{-1} A D=\beta I+\sqrt{\alpha \gamma} B
$$


has the same eigenvalues $\lambda_{j}$ as $A$ in (31). The ansatz for an eigenvector $z$ of $B$ is

$$
z_{j}=u \xi_{1}^{j}+v \xi_{2}^{j}, j=1, \ldots n
$$

For $j<n$, the difference equation for $z_{j}$ is

$$
z_{j+1}-\lambda z_{j}+z_{j-1}=0 .
$$

Thus, $\xi_{1}$ and $\xi_{2}$ are the roots of

$$
\xi_{k}^{2}-\lambda \xi_{k}+1=0, k=1,2,
$$

and

$$
\lambda=\xi_{1}+\xi_{1}^{-1}=\xi_{2}+\xi_{2}^{-1} .
$$

At $j=0$

$$
z_{0}=u+v=0,
$$

and, since $\xi_{2}=1 / \xi_{1}$, we have at $j=n$

$$
\begin{aligned}
& u\left(\left(a_{0}-\lambda\right) \xi_{1}^{n}+a_{1} \xi_{1}^{n-1}+\ldots+a_{m} \xi_{1}^{n-m}\right)+ \\
& v\left(\left(a_{0}-\lambda\right) \xi_{1}^{-n}+a_{1} \xi_{1}^{-n+1}+\ldots+a_{m} \xi_{1}^{-n+m}\right)=0 .
\end{aligned}
$$

Then we conclude from (35), (36), and (37) that $\xi_{1}$ satisfies

$$
\xi_{1}^{2 n-2 m} g\left(\xi_{1}\right)=g\left(\xi_{1}^{-1}\right),
$$

with $g$ defined in (32). If $g$ has the roots $\xi_{j}, j=1, \ldots m+1$, then (38) can be rewritten as (33). The lemma follows from (35) and the spectral equivalence between $D^{-1} A D$ and $A$.

Using the lemma we can prove the following theorem:

Theorem 4.2 The eigenvalues of $C$ and $V$ in (23) satisfy

$$
\Re \lambda(C)>0, \quad \Re \lambda(V)<0 .
$$

Proof Firstly, let $A$ in lemma 4.1 be equal to $C$. Then $m=2, \alpha=-1, \beta=$ $0, \gamma=1, \rho=i, \sqrt{\alpha \gamma}=i, \kappa_{0}=3, \kappa_{1}=-4$, and $\kappa_{2}=1$. Furthermore, $a_{0}=$ $-3 i, a_{1}=4$, and $a_{2}=i$. Hence,

$$
g(\xi)=-(\xi+i)^{3}
$$

and the equation corresponding to (33) is

$$
-\xi^{2 n-1}(\xi+i)^{3}=i(\xi-i)^{3} .
$$




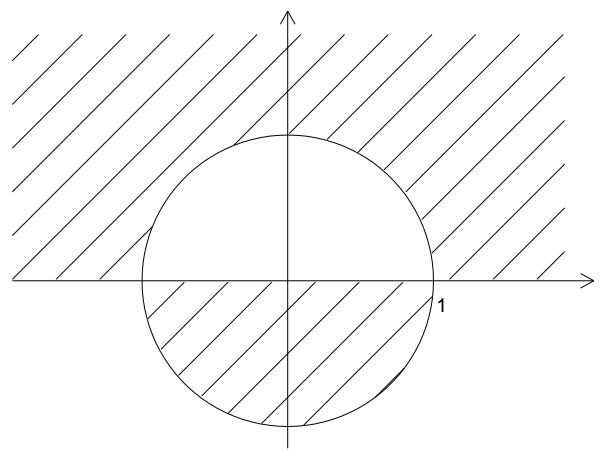

(a)

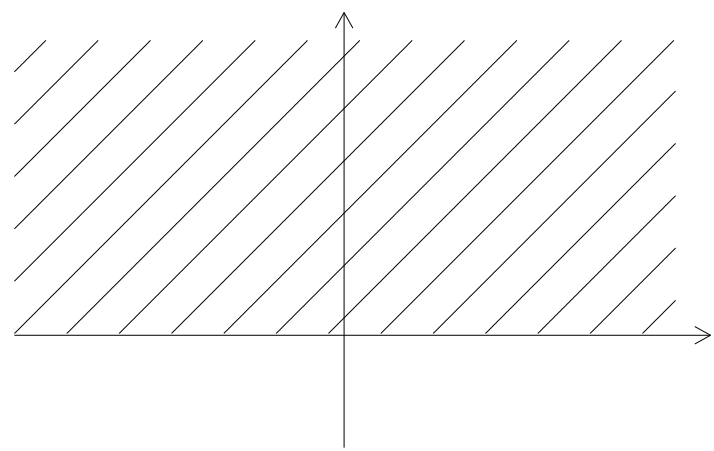

(b)

Figure 4: The mapping of the roots of (33) in (a) to the eigenvalues of $B$ in (b).

Let $\xi=r \exp (i \phi)$ and take the modulus of the left and the right hand sides of (39) to obtain

$$
r^{2 n-1}=|\xi-i|^{3} /|\xi+i|^{3} .
$$

On the real axis except for $\xi \pm 1$, the left hand side lhs in (40) is less than 1 inside the circle and $l h s>1$ outside the circle of radius 1 in Fig 4.a, while the right hand side $r h s=1$. The roots $\xi \pm 1$ are double roots of (34) with $\lambda= \pm 2$. Then $z_{j}=\xi^{j}(u+v)$ but $u+v=0$ in (36). Since $z_{j}=0$ for all $j$ is not an eigenvector, $\lambda= \pm 2$ is not an eigenvalue of $B$, and there is no $\xi$ on the real axis corresponding to an eigenvalue $\lambda(B)$.

For $r \leq 1$ and $\phi \in(-\pi, 0)$, lhs in (40) satisfies lhs $\leq 1$, but $r h s>1$. Hence, there is no solution in the closed shaded sector of the circle in Fig 4.a. For $r \geq 1$ and $\phi \in(0, \pi)$, lhs $\geq 1$ but $r h s<1$ and again there is no solution to (39). The shaded area in the upper half plane in Fig 4.a has no solution. The shaded domain in Fig 4.a is mapped to the upper half plane including the real axis in Fig 4.b by $\xi+\xi^{-1}$. Therefore the eigenvalues of $C$ fulfill

$$
\lambda(C)=\beta+\sqrt{\alpha \gamma}\left(\xi+\xi^{-1}\right)=i(x-i y)=y+i x,
$$

for some $x$ and $y>0$, i.e. $\Re \lambda(C)>0$.

Secondly, let $A$ in lemma 4.1 be equal to $V$. Then $m=3, \alpha=1, \beta=-2, \gamma=$ $1, \rho=1, \sqrt{\alpha \gamma}=1, \kappa_{0}=2, \kappa_{1}=-5, \kappa_{2}=4$, and $\kappa_{3}=-1$. Here,

$$
g(\xi)=-(\xi-1)^{4}
$$

and the root equation (33) is

$$
\xi^{2 n-2}(\xi-1)^{4}=(\xi-1)^{4} \Rightarrow \xi^{2 n-2}=1
$$


The root $\xi=1$ is not a root of (41) corresponding to an eigenvalue of $B$ for the same reason as above. The other roots have the form $\xi_{j}=\exp \left(i \phi_{j}\right), \phi_{j} \neq 0$. Thus, the eigenvalues of $V$ are

$$
\lambda(V)=\beta+\sqrt{\alpha \gamma}\left(\xi_{j}+\xi_{j}^{-1}\right)=-2+\exp \left(i \phi_{j}\right)+\exp \left(-i \phi_{j}\right)=-2+2 \cos \left(\phi_{j}\right)<0,
$$

and the theorem is proved.

It is in principle possible to estimate the eigenvalues of $S$ via lemma 4.1 when $\mu>0$ and $\theta \neq \pi / 2$, but it would be rather tricky. Instead they are evaluated numerically. In Fig. 5.a the smallest and largest real part of the eigenvalues of $0.5 C-\nu V$ when $n_{x}=50$ are plotted for different $\nu$-values. All eigenvalues have a positive real part. Multiplied by a scaling parameter, they are the same as those of $S$ for some $\nu$.

The system matrix $U$ for the upwind scheme with the stencil $S_{u+}^{c}$ in (6) has the non-zero elements

$$
U_{j j}=3 / 2, U_{j, j-1}=-2, j \geq 2, U_{j, j-2}=1 / 2, j \geq 3 .
$$

It is defective and has one eigenvalue $\lambda_{j}(U)=3 / 2$. If viscosity is added then $U-\nu V$ has a complete eigenvector system. The real part of the eigenvalues of $U-\nu V$ is also positive in Fig. 5.b.

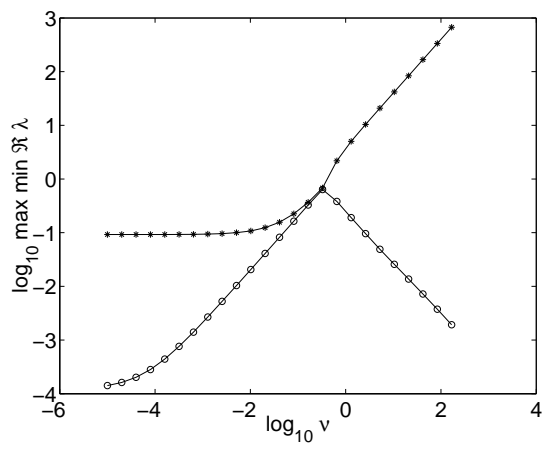

(a)

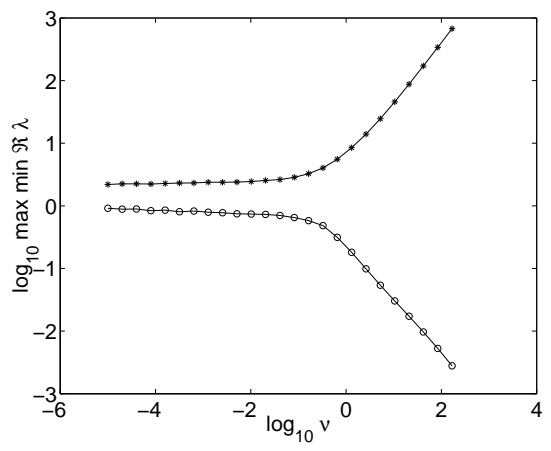

(b)

Figure 5: Maximum and minimum of the real part of the eigenvalues of $0.5 C-\nu \mathrm{V}$ (a) and of $U-\nu V$ (b) for different $\nu$.

Consider now centered differencies $S_{c}^{c}$ for the convection in space also at the interface, and construct the corresponding matrix $R$. We use the symmetric interpolation operator $H_{7}^{*}$ at the block interface. In this case both $R_{l r}$ and $R_{r l}$ are non-zero and an analysis is complicated. The eigenvalues are also here computed numerically on a domain partitioned into a left block with $8 \times 8$ cells and a right block with $16 \times 16$ cells. The largest real part of the eigenvalues of $-R$ is computed and plotted in Fig. 6, so that the Figures indicate stability when 
$-\Re \lambda_{j}(R)<0$. The result is highly dependent on the direction of convection $\theta$. In Fig. 6.a, a diffusion term introduces the necessary damping with $\mu \neq 0$ and in Fig. 6.b we use artificial dissipation $\eta_{i j}$ in (7). There is always an eigenvalue $\lambda=0$ with eigenvector $w=x$ for $\theta=\pi / 2$. The results for $\theta \neq \pi / 2$ move downwards if we increase the dissipation, and upwards if we decrease it. No interpolation operator in Table 1 is stable without dissipation $\sigma$ or diffusion $\mu$.

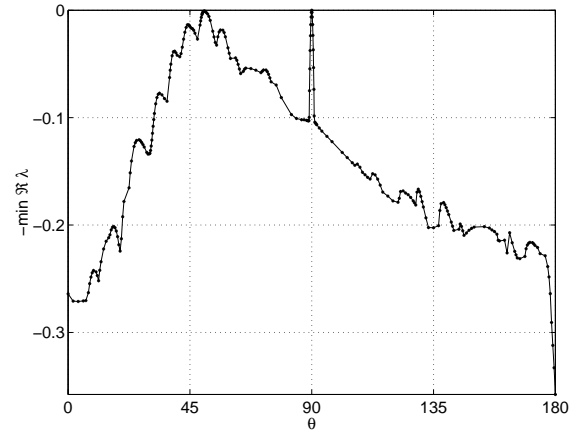

(a)

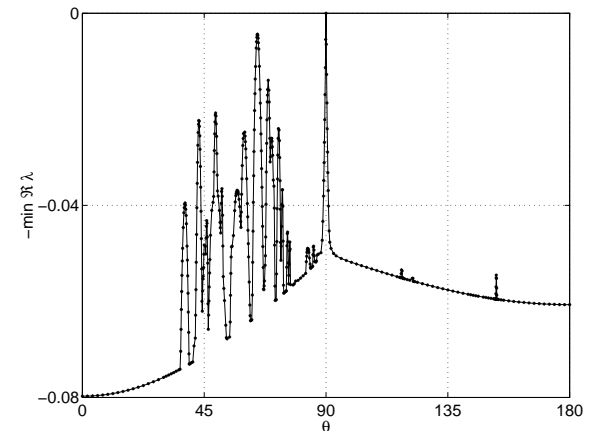

(b)

Figure 6: Largest real part of the eigenvalues of $-R$ for $0^{\circ} \leq \theta \leq 180^{\circ}$ and diffusion with $\mu=7.5 \cdot 10^{-4}$ (a) and for artificial dissipation with $\sigma=0.012$ (b).

The conclusion is that with the upwind discretization at least at the block interface the discretization is stable if the interpolation in the ghost cells also is one-sided there. Either diffusion in the equation or added artificial dissipation is necessary for the centered scheme. The amount of dissipation needed for different interpolation operators is compared for Navier-Stokes' equations in Section 5.

\section{Navier-Stokes' equation}

We found in Sect. 4 that the interpolation operators in Sect. 3 had different stability properties when they were used together with the centered difference approximation $S_{c}^{c}$ in (6) for the convection-diffusion equation (2). The same interpolation schemes are here implemented in a code for computing the steady state solution of the compressible Navier-Stokes equations in two dimensions.

Let $\rho$ be the density, $u$ and $v$ the velocity components, $E$ the total energy, and $p$ the pressure. Then the flux functions $f$ and $g$ in (1) for Navier-Stokes' equation corresponding to $w=(\rho, \rho u, \rho v, \rho E)^{T}$ are

$$
f=\left(\begin{array}{c}
\rho u \\
\rho u^{2}+p-\mu S_{11} \\
\rho u v-\mu S_{21} \\
(\rho E+p) u-\mu\left(u S_{11}+v S_{21}\right)
\end{array}\right), g=\left(\begin{array}{c}
\rho v \\
\rho u v-\mu S_{12} \\
\rho v^{2}+p-\mu S_{22} \\
(\rho E+p) v-\mu\left(u S_{12}+v S_{22}\right)
\end{array}\right)
$$


where $S$ is the strain rate tensor defined by

$$
S=\left(\begin{array}{cc}
\frac{4}{3} u_{x}-\frac{2}{3} v_{y} & u_{y}+v_{x} \\
u_{y}+v_{x} & \frac{4}{3} v_{y}-\frac{2}{3} u_{x}
\end{array}\right) .
$$

The relation between $E, p$, the gas constant $\gamma$ and the other variables is

$$
E=p /((\gamma-1) \rho)+0.5\left(u^{2}+v^{2}\right)
$$

A grid is generated and partitioned into blocks. When the grid size is reduced in a block then it is halved in both directions. The grid is intentionally slightly skewed to avoid a Cartesian grid, see Fig. 7. The intention here is not to compute an accurate solution but to evaluate the stability of the interpolation operators.

The convective terms are discretized by the Jameson scheme [13] and the viscous terms by the centered approximation in Sect. 2. A three-stage RungeKutta scheme with three levels of multigrid iteration is used to compute the steady state solution as in [7]. In the computations, the Mach number is 0.5 and the Reynolds number $R e=\mu^{-1}=5000$. The code is validated by comparison to the Blasius solution [19] in Fig 7.b.

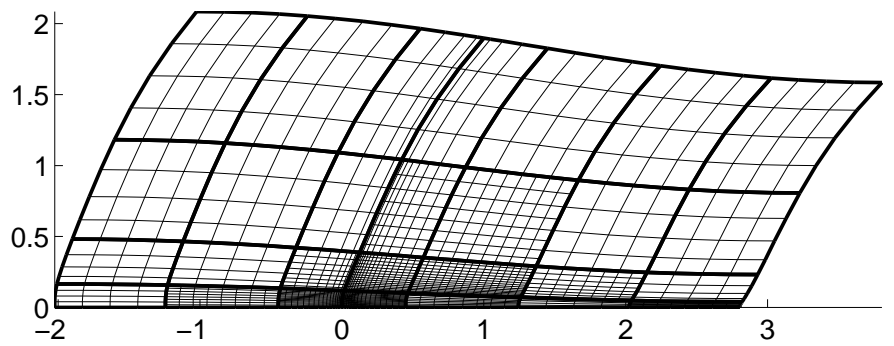

(a)

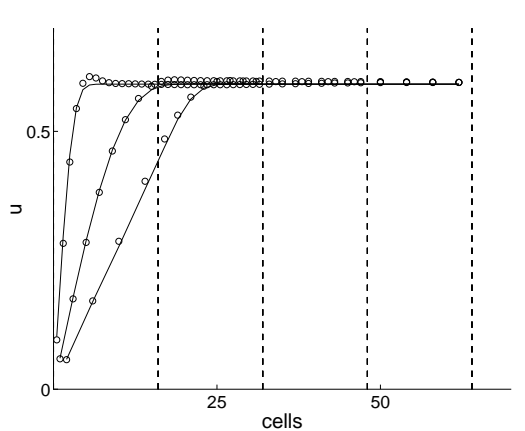

(b)

Figure 7: (a) The grid over the flat plate at $x \geq 0, y=0$. (b) The velocity component $u$ at three stations in the middle of the first three blocks after $x=0$ above the flat plate for the Blasius solution (solid) and the computed solution (o). The velocities are examined in one column of cells in the grid.

The stability performance of the interpolation operators in Table 1 is tested on this grid. The solution is computed with fixed artificial dissipation with $\sigma=0.02$ in (7). Table 2 contains the number of iterations needed to reduce the norm of the residual by five decades. The symbol ' $x$ ' means that the solution diverges or that it fails to converge within 1000 iterations. The symbol '-' in columns for symmetrized operators is used to avoid repetition since some operators have identical symmetric versions. 


\begin{tabular}{|c|c|c|c|c|c|}
\hline & & \multicolumn{2}{|c|}{ unsymmetric } & \multicolumn{2}{c|}{ symmetric } \\
\hline & order & $\sigma$ & $M$ & $\sigma$ & $M$ \\
\hline$L_{1}$ & 3 & .0131 & 229 & .0150 & 229 \\
\hline$L_{2}$ & 3 & .0253 & $\mathrm{x}$ & .0286 & $\mathrm{x}$ \\
\hline$H_{1}$ & 4 & .0334 & $\mathrm{x}$ & .0389 & $\mathrm{x}$ \\
\hline$H_{2}$ & 4 & .0390 & $\mathrm{x}$ & - & - \\
\hline$H_{3}$ & 4 & .0311 & $\mathrm{x}$ & - & - \\
\hline$H_{4}$ & 4 & .0168 & $\mathrm{x}$ & .0209 & 280 \\
\hline$H_{5}$ & 4 & .0176 & 236 & - & - \\
\hline$H_{6}$ & 4 & .0157 & 244 & .0188 & 312 \\
\hline$H_{7}$ & 4 & .0119 & 214 & .0116 & 214 \\
\hline
\end{tabular}

Table 2: Estimates of the required amount of dissipation $\sigma$ for stability and observed number of iterations for convergence $M$.

The eigenvalues of $-R$ are computed as in Fig. 6 for the model equation (2) with $\mu=0$. Then artificial dissipation $\eta$ is added so that $-\Re \lambda_{j}(R)<0$ for all $\theta$. The minimum coefficient $\sigma$ for stability is shown in Table 2. It follows from the Table that the iterations do not converge when large dissipation $\sigma>$ 0.02 is needed for stability in the eigenvalue analysis of the model equation. Considering the efficiency and simplicity of the symmetric operators and the stability analysis, we recommend the symmetric version of operator $H_{7}$ when fourth order interpolation is required.

\section{Conclusion}

The computational grid is partitioned into a number of blocks. The grid size may jump at grid interfaces by a factor two. For second order accuracy in the discretization of second derivatives, fourth order accurate interpolation is required at the interfaces. Upwind approximations are stable at the interface but centered difference discretizations need extra artificial dissipation or diffusion in the equation to be stable.

\section{References}

[1] M. Berger, Stability at interfaces with mesh refinement, Math. Comput., 45 (1985), pp. 301-318.

[2] M. Berger, On conservation at grid interfaces, SIAM J. Numer. Anal., 24 (1987), pp. 967-984. 
[3] M. Berger, P. Colella, Local adaptive mesh refinement for shock hydrodynamics, J. Comput. Phys., 82 (1989), pp. 64-84.

[4] B. Cathers, S. Bates, Spurious numerical refraction, Int. J. Numer. Meth. Fluids, 21 (1995), pp. 1049-1066.

[5] B. Cathers, S. Bates, B. A. O'Connor, Internal wave reflections and transmissions arising from a non-uniform mesh. Part I: An analysis for the Crank-Nicholson linear finite element scheme, Int. J. Numer. Meth. Fluids, 9 (1989), pp. 783-810.

[6] P. Colella, M. R. Dorr, D. D. Wake, Numerical solution of plasma fluid equations using locally refined grids, J. Comput. Phys., 152 (1999), pp. $550-583$.

[7] L. Ferm, P. Lötstedt, Blockwise adaptive grids with multigrid acceleration for compressible flow, AIAA J., 37 (1999), 121-123.

[8] L. Ferm, P. Lötstedt, Adaptive error control for steady state solutions of inviscid flow, SIAM J. Sci. Comput., 23 (2002), 1777-1798.

[9] B. Gustafsson, H.-O. Kreiss, J. Oliger, Time Dependent Problems and Difference Methods, Wiley, New York, 1995.

[10] R. D. Hornung, J. A. Trangenstein, Adaptive mesh refinement and multilevel iteration for flow in porous media, J. Comput. Phys., 136 (1997), pp. 522-545.

[11] I. S. Kim, W. J. R. Hoefer, A local mesh refinement algorithm for the time-domain finite-difference method using Maxwell's curl equations, IEEE Trans. Microwave Theory Techn., 38 (1990), pp. 812-815.

[12] P. Lötstedt, S. Söderberg, A. Ramage, L. HemmingssonFRÄNDÉN, Implicit solution of hyperbolic equations with space-time adaptivity, BIT, 42 (2002), 134-158.

[13] A. Jameson, W. Schmidt, E. Turkel, Numerical solutions of the Euler equations by finite volume methods using Runge-Kutta time-stepping schemes, AIAA-paper 81-1259, 1981.

[14] M. Okoniewski, E. Okoniewski, M. A. Stuchly, Three-dimensional subgridding algorithm for FDTD, IEEE Trans. Ant. Prop., 45 (1997), pp. $422-429$.

[15] K. Отто, Analysis of preconditioners for hyperbolic partial differential equations, SIAM J. Numer. Anal., 33 (1996), pp. 2131-2165. 
[16] E. PÄrt-Enander, B. SjÖGreen, Conservative and non-conservative interpolation between overlapping grids for finite volume solutions of hyperbolic problems, Computers \& Fluids, 23 (1994), pp. 551-574.

[17] D. T. Prescott, N. V. Shuley, A method for incorporating different sized cells into the finite-difference time-domain analysis technique, IEEE Microwave Guided Wave Lett., 2 (1992), pp. 434-436.

[18] K. G. Powell, P. L. Roe, T. J. Linde, T. I. Gombosi, D. L. De ZEEUW, A solution adaptive upwind scheme for ideal magnetohydrodynamics, J. Comput. Phys., 154 (1999), pp. 284-309.

[19] H. Schlichting, Boundary-Layer Theory, 7th ed., McGraw-Hill, New York, 1979.

[20] P. Wesseling, Principles of Computational Fluid Dynamics, Springer, Berlin, 2001.

[21] S. S. Zivanovic, K. S. Yee, K. K. Mei, A subgridding method for the time-domain finite-difference method to solve Maxwell's equations, IEEE Trans. Microwave Theory Techn., 39 (1991), pp. 471-479. 